\title{
Socioeconomic variation in hysterectomy up to age 52: national, population based, prospective cohort study
}

\author{
Sarah F Marshall, Rebecca J Hardy, Diana Kuh
}

We have previously reported striking inverse social gradients in the risk of hysterectomy in a large national cohort of women born in England, Scotland, or Wales followed since their birth in March 1946 until the age of 43 years. ${ }^{1}$ This cohort has now been followed to age 52 years, and the cumulative hysterectomy risk has doubled (from $10 \%$ to $21 \%$ ). We examined whether the social gradient in hysterectomy attenuated among women between the ages of 43 and 52 years.

\section{Participants, methods, and results}

Of the 2547 women in the Medical Research Council's national survey of health and development, 1755 (69\%) provided information on gynaecological surgery. Losses to follow up were due to death (157), emigration (232), refusal to participate (234), or failure to be traced (169). Socioeconomic status was measured as before, ${ }^{1}$ using highest educational qualifications and partner's and own social class. Kaplan-Meier survivor function estimates were plotted to compare the survival distributions (time until hysterectomy) by each indicator of socioeconomic status, and Cox's regression models with time dependent covariates were used to examine the gradient in risk of hysterectomy by these factors. Social class was fitted as a linear trend, with social class $\mathrm{V}$ as the baseline and education as a categorical factor, with no qualifications as baseline.

Women with less education were more likely to have had a hysterectomy by the age of 52 (Breslow statistic 15.05, $\mathrm{df}=4 ; \mathrm{P}=0.005$ ) (figure). As at age 43 years, the highest cumulative risk was for women with minimal qualifications (below O level) (28\% women had had a hysterectomy by the age of 52), and the lowest was for the best educated women (12\%). By age 52, similar risks were observed for women with no qualifications (21\%), those with O levels (21\%), and those with A levels (18\%). The proportional hazards assumption did not hold; the hazard ratio for women in the highest three educational categories, compared with women with no qualifications, attenuated with increasing age.

A Cox's proportional hazard model with follow up until age 43 years confirmed the inverse gradient with partner's social class (hazard ratio 0.85 (95\% confidence interval 0.75 to 0.95$)$ ) found previously. ${ }^{1}$ Similar modelling for the updated follow up showed a weaker gradient (0.93 (0.86 to 1.01$)$ ). As the hazard ratio decreased with age, a piecewise Cox's regression model was fitted with two time periods, up to and including 43 years and 44-52 years. The hazard ratios for these two age periods differed $(\mathrm{P}=0.08)$, with a lack of a social class gradient between 44-52 years (1.01 (0.89 to 1.15)). Adjustment for parity, obesity, and prior sterilisation-factors that may be associated with risk of hysterectomy-attenuated but did not abolish the excess hazard up to age 43 . The effect of own social class was of marginal significance during both time periods.

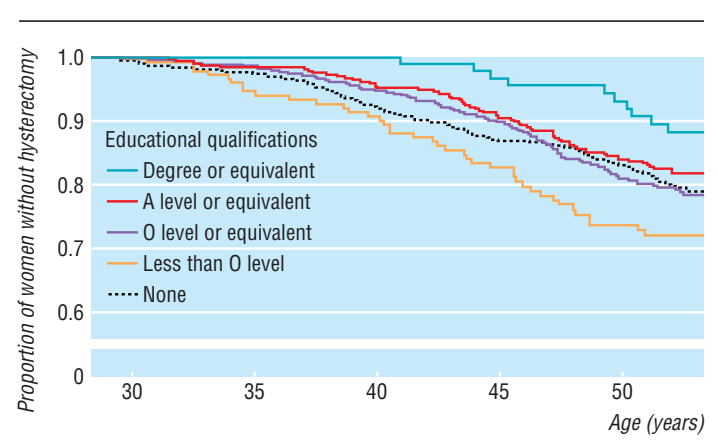

Kaplan-Meier survival plot for age at hysterectomy by education

\section{Comment}

The inverse social gradient in hysterectomy has been observed in several studies, ${ }^{2}{ }^{3}$ but this study is the first to report changing effects over time. Despite the attenuation in the social gradient in terms of hazard ratio, the most educated women still had a lower cumulative risk of hysterectomy than their peers. Hysterectomies for benign diagnoses, when the decision to operate may be more influenced by social factors, tend to be carried out at a younger age than those for cancer, when the decision is more likely to be made on medical grounds. ${ }^{3}$ Hysterectomies for menstrual bleeding, for example, have been shown to be inversely related to social class ${ }^{4}$ and education ${ }^{5}$ and have become more common at younger ages. This could account for the greater social differentials in hysterectomy at younger rather than older ages. Alternatively, the diseases and conditions for which hysterectomy provides a treatment may occur later in women from higher social groups.

Contributors: SFM prepared the data, conducted the analyses, and drafted the paper. RJH supervised the analysis, interpreted the results, and substantially revised the text. DK coordinated the data collection for the women's health study, had the original idea for the paper, and jointly planned the analysis, drafted the paper, and revised the text. All three authors act as guarantors.

Funding: Medical Research Council.

Competing interests: None declared.

1 Kuh D, Stirling S. Socioeconomic variation in admission for diseases of female genital system and breast in a national cohort aged 15-43. BMJ 1995;311:840-3.

2 Kjerulff K, Langenberg P, Guzinski G. The socioeconomic correlates of hysterectomies in the United States. Am J Public Health 1993;83:106-8.

3 Settnes A, Jorgensen T. Hysterectomy in a Danish cohort. Prevalence, incidence and socio-demographic characteristics. Acta Obstet Gynecol Scand 1996;75:274-80.

4 Vessey MP, Villard-Mackintosh L, McPherson K, Coulter A, Yeates D. The epidemiology of hysterectomy: findings in a large cohort study. BrJ Obstet Gynaecol 1992;99:402-7.

5 Brett KM, Marsh JVR, Madans JH. Epidemiology of hysterectomy in the United States: demographic and reproductive factors in a nationally representative sample.J Women's Health 1997;6:309-16.

(Accepted 15 March 2000)
Medical Research Council National Survey of Health and Development, Department of Epidemiology and Public Health, Royal Free and University College London Medical School, London

WC1E 6BT

Sarah F Marshall research scientist Rebecca J Hardy research scientist Diana Kuh senior research scientist

Correspondence to: D Kuh d.kuh@ucl.ac.uk

BMJ 2000;320:1579 